KCNN3, LGI4, EGFLAM, HPGDS and four lncRNAs (LINCHOXD1-1, LINC-MLN-5, LINC-RPIA-2, LINC-LY86-4). For $J A K 2$ and KCNN3 genes, we found in literature the evidence of their potential role in resistance of ovarian cancer.

Conclusion* On the basis of transcriptome profile, we discovered till unknown associations of gene expression levels with response in ovarian carcinoma patients (MYH11, SETDB2, IRAG2, FOXP2 and LGI4) together with four lncRNAs (LINC-HOXD1-1, LINC-MLN-5，LINC-RPIA-2，LINC-LY864) with potential role in therapy resistance in ovarian carcinoma patients.

\section{PRECLINICAL STUDIES SUPPORT THERAPEUTIC APPLICATION OF THE LEUKAEMIC CELL-BASED CANCER RELAPSE VACCINE DCP-001 IN OVARIAN CANCER}

${ }^{1} \mathrm{M}$ Nagasawa, ${ }^{1} \mathrm{R}$ Bos, ${ }^{1} \mathrm{H}$ Zuo, ${ }^{2} \mathrm{KYHW}$ Yin, ${ }^{1} \mathrm{MJ}$ Van Lierop, ${ }^{2} \mathrm{~S}$ Tabruyn, ${ }^{1} \mathrm{E}$ Manting ${ }^{3} \mathrm{~A}$ Vledder, ${ }^{3} \mathrm{M}$ De Bruyn, ${ }^{3} \mathrm{H}$ Nijman, ${ }^{1} \mathrm{SK}$ Singh*. ${ }^{1}$ Immunicum $A B$, RandD, Leiden, Netherlands; ${ }^{2}$ TransCure BioServices, France; ${ }^{3}$ University Medical Center Groningen , Obstetrics and Gynecology, Groningen, Netherlands

\subsection{6/ijgc-2021-ESG0.449}

Introduction/Background* Ovarian cancer (OC) causes high mortality due to late diagnosis and high rate of relapse following initial therapy. Immunotherapy in combination with standard treatment modalities forms a promising new treatment approach.

DCP-001 is an intradermally applied cancer relapse vaccine derived from the human leukaemic cell line DCOne ${ }^{\circledR}$ and is currently tested in acute myeloid leukaemia patients. To obtain the highly immunogenic DCP-001 vaccine, DCOne cells are shifted towards a mature dendritic cell phenotype. Since DCOne cells express multiple common tumour associated antigens such as WT-1, RHAMM, PRAME and MUC-1, which are also documented as target antigens in OC, DCP-001 vaccination may also be efficacious in OC. To support this hypothesis, the capacity of DCP-001 to induce immune responses against OC was studied in human peripheral blood mononuclear cells (PBMCs) from OC patients and a humanized mouse model for OC.

Methodology The effect of DCP-001 on T cells was evaluated after a 3 week culture of PBMCs with or without DCP-001. Cytotoxic activity was analysed by IFN $\gamma$ production and CD107a expression when these cells were subsequently cultured with OC cell line SKOV3. The effect of DCP-001 vaccination in vivo was evaluated in humanised NCG mouse subcutaneously engrafted with SKOV3 OC cells. Mice received two intra-peritoneal (i.p.) vaccinations with DCP-001 either after or prior to SKOV3 engraftment and tumour size was measured to evaluate the efficacy of DCP-001.

Result(s)* In vitro, DCP-001 was shown to activate both $\mathrm{CD}^{+}$as well as $\mathrm{CD}^{+} \mathrm{T}$ cells and to induce formation of memory $\mathrm{T}$ cells. Importantly, DCP-001-stimulated $\mathrm{CD}^{+} \mathrm{T}$ cells from OC patients were shown to exert a HLA class I dependent, immune response to OC cells. In vivo, in an ovarian tumour mouse model, significant reduction of tumour growth rate and partial or even complete tumour regressions were observed in mice vaccinated with DCP-001, particularly when administered as relapse vaccine (prior to tumour engraftment), as compared to PBS treated mice.

Conclusion* These pre-clinical in vitro and in vivo results support the potential use of DCP-001 as a cancer relapse vaccine in ovarian cancer, with the aim to reduce disease recurrence following initial standard of care therapy.

\section{EVALUATION OF THE LOX GENE/PROTEIN AS POTENTIAL PROGNOSTIC MARKER IN OVARIAN CANCER}

${ }^{1}$ JP Syrkis, ${ }^{1}$ KA Kujawa, ${ }^{2}$ E Zembala-Nożyńska, ${ }^{3} \mathrm{AJ}$ Cortez* ${ }^{*}{ }^{4} \mathrm{~J}$ Kupryjańczyk, ${ }^{1} \mathrm{KM}$ Lisowska. ${ }^{1}$ Maria Sklodowska - Curie National Research Institute of Oncology, Gliwice Branch, Center for Translational Research and Molecular Biology of Cancer, Gliwice, Poland; ' 2 Maria Sklodowska - Curie National Research Institute of Oncology, Gliwice Branch, Tumor Pathology Department, Gliwice, Poland; ${ }^{3}$ Maria Sklodowska - Curie National Research Institute of Oncology, Gliwice Branch, Department of Biostatistics and Bioinformatics, Gliwice, Poland; ${ }^{4}$ Maria Sklodowska - Curie National Research Institute of Oncology, Tumor Pathology Department, Warszawa, Poland

\subsection{6/ijgc-2021-ESG0.450}

Introduction/Background* In our previous microarray study we identified a set of genes (multigene signature) related with different survival of patients with high-grade serous ovarian cancer (HG-SOC) [1]. One of the genes from this signature was LOX, encoding for lysyl oxidase. LOX is engaged in the cross-linking of the extracellular matrix proteins. Various studies have indicated that LOX may act as either an oncogene or a tumor suppressor, depending on the type of tissue/tumor. As our previous study indicated that higher LOX mRNA level was negatively correlated with survival of ovarian cancer patients, we aimed to check whether LOX level determined by immunohistochemistry shows the same dependency and could be useful for clinical practice.

Methodology Two commercially available anti-LOX antibodies (ab174316-Abcam, NB-2530-Novus Biologicals) were validated by western blotting, using protein extracts from six ovarian cancer cell lines and recombinant LOX protein as a control (NBP-59887-Novus Biologicals). Then, immunohistochemical analysis was performed (tissue samples of HG-SOC) and on tissue arrays containing spectrum of different histological type, FIGO stages, etc. (US Biomax). The results were analyzed using Statistica (version-13.1, StatSoft Poland).

Result(s)* Only one antibody (ab174316-Abcam) was positively validated as specific toward LOX and it was used for further analysis. Unfortunately, Kaplan-Meier analysis showed no correlation between LOX protein level and the patients' survival time. Further analysis revealed that LOX level was correlated with primary/metastatic tumor difference: higher stromal LOX expression occurred eight times more often in metastatic than in primary tumors. However, there was no such correlation when LOX expression was assessed in cancer cells.

Conclusion* Although our previous observations indicated that higher LOX mRNA level was correlated with shorter survival of ovarian cancer patients, protein level of LOX does not demonstrate prognostic value in the analyzed group of patients with HG-SOC. However, we observed significantly higher LOX expression in the stroma of metastases compared to that of primary tumors. This observation is consistent with the assumption that LOX is associated with a more aggressive tumor phenotype.

[1] Katarzyna Lisowska et al. doi: 10.1007/s00432-016 2147-y

Acknowledgments This study was supported from grant Miniatura 3 (2019/03/X/NZ5/00564) from National Science Center. A.J.C. was co-financed by the European Union through the European Social Fund (grant no. POWR.03.02.00-00-I029) 